\title{
Implementation of both Spatial Diversity and Spatial Multiplexing Technique in MIMO-OFDMA Communication System
}

\begin{abstract}
Babidha $\mathrm{K} \mathrm{R}^{1}$ and Smitha $\mathrm{P} \mathrm{S}^{2}$
Student, Dept of ECE, SCT College of Engineering, Trivandrum, Kerala, India, babidha@gmail.com ${ }^{1}$

Assistant Professor, Dept of ECE, SCT College of Engineering, Trivandarum, Kerala, India smitha.krishnendu@gmail.com ${ }^{2}$

Abstract

MIMO (Multiple Input Multiple Output) that use multiple antennas in transmitter and receiver is considered to be powerful technology in future wineles communication system. The major problem facing in this multipath propagation is the interference between symbols transmitted through adjacent channels. $i$. e Inter Symbol Interference. This could be avoided if we use OFDMA (Orthogonal Frequenct Division Multiple Access) technology. Thus MIMO combined with OFDM has got prior thportance in future wireless communication system. The major properties of MIMO system involve Spaticl Diversity and Spatial Multiplexing. In former case same copies of data is being transmitted in the MIMO channels while in latter case different user's data is being transmitted in the MIMO channels. In this paper, a MIMOOFDMA system with both the advantages of Spatial Diversity and Spatial Multiplexing is suggested.
\end{abstract}

Keywords: MIMO, OFDMA Spatial Multiplexing, Spatial Diversity, Inter Symbol Interference

\section{Introduction}

MIMO (Multiple Input Multiple Output) system using multiple antennas in both transmitter and receiver of a communication system is able to achieve high data rates and better performance oompared to SISO (Single Input Single Output) systems. Here the MIMO channel will be divided into multiple sub channels and data will be transmitted through those channels [2 3, 8]. The major issue while transmitting symbols though adjacent channels is the interference between those symbols leading to Inter Symbol Interference (ISI). In order to avoid this Inter Symbol Interference (ISI), OFDMA technology is adopted in MIMO systems. Thus MIMO combined with OFDMA has become a powerful technology in future wireless communication system with high data rates and better performance that eliminates Inter Symbol Interference (ISI).

The major properties or subclasses of MIMO system are Spatial Diversity and Spatial Multiplexing. In Spatial Diversity same copies of data from single user will be transmitted through all sub channels. On the other hand in Spatial Multiplexing, different users will be sending data through the sub channels. In Spatial Diversity technique the error rate of retrieved copies will be pretty much less due to multiple copies of data [3]. Redundancies in received data reduce the error rate. In spatial multiplexing technique, since different users send data through MIMO channel the throughput and capacity of the system improves substantially [2-3]. 
Thus implementing both spatial Diversity and Spatial Multiplexing in MIMO-OFDMA technology are discussed in this paper. The performance and capacity analysis is investigated as a function of SNR of the MIMO channel. In section II description of the 8X8 MIMO system designed implementing both Spatial Diversity and Spatial Multiplexing is provided. In section III Simulation results of the performance and capacity analysis are provided.

\section{System Model}

General system model for MIMO communication system will consist of $\mathrm{N}_{\mathrm{t}}$ Transmitter antennas and $\mathrm{N}_{\mathrm{r}}$ Receiver antennas. In this paper 8 X 8 MIMO system is considered with 4 users sharing the MIMO channel. All the users are allowed to send data through MiMO channel at same time slot.

\section{Transmitter Model}

Here a MIMO OFDMA communication with Nt Transmitter antennas and Nr Receiver antennas is considered. Each user will be ending data through MIMO channel simultaneously. Let each user's message bits be

$$
\mathbf{B}=\left\{\mathbf{b}_{0}, \mathbf{b}_{1}, \mathbf{b}_{2}, \mathbf{b}_{3} \ldots \mathbf{b}_{\mathrm{N}-1}\right\}
$$

This input message sequence of each userwill be mapped into BPSK modulator. BPSK modulator is chosen since it is the simplest form of phase shift keying modulation. This modulation is said to be most robust sinee it takes the highest level of noise distortion to make the demodulator reach in an incorrectedecision. Let BPSK modulated symbols be

$$
\mathrm{S}=\left\{\mathrm{s}_{0}, \mathrm{~S}_{1}, \mathrm{~s}_{2}, \mathrm{~s}_{3}, \ldots \mathrm{s}_{\mathrm{N}-1}\right\}
$$

And these modulated symbols are allowedtg pass through STBC encoder which encodes BPSK symbols into $\mathrm{Nr}$ sequence of data

$$
\mathrm{C}_{\mathrm{k}}=\left\{\mathrm{c}_{\mathrm{k}}^{(0)}, \mathrm{c}_{\mathrm{k}}^{(1)}, \mathrm{c}_{\mathrm{k}}^{(2)}, \ldots \ldots . \mathrm{c}_{\mathrm{k}}^{(\mathrm{N}-1)} \mathrm{r}\right\}
$$

where $\mathrm{C}_{\mathrm{k}}{ }^{(\mathrm{i})}$ is the coded symbols transmitted from $\mathrm{i}^{\text {th }}$ antenna to the $\mathrm{k}^{\text {th }}$ sub channel in MIMO system. Transmission matrix for two transmit antenna using Alamounti code

$$
\bigcirc \mathbf{G}=\left[\begin{array}{cc}
c_{1} & c_{2} \\
-c_{1}^{*} & -c_{2}^{*}
\end{array}\right]
$$

The matrix mentioned aboye is for two transmitter antennas using Alamounti's code. Here in above matrix, numben of columns denotes number of transmitter antennas and number of row denotes time slot at which data is being transmitted through MIMO channel. Thus for a 2X2 MIMO system at first time slot $\mathrm{c}_{1}$ and $\mathrm{c}_{2}$ will be transmitted through antenna 1 and antenna 2 respectively and simultaneously. At second time slot $-\mathrm{c}_{2}{ }^{*}$ and $\mathrm{c}_{1}{ }^{*}$ will be transmitted from antennas 1 and 2 simultaneously. The encoded data is appended with pilot bits in the beginning. The pilot bits are known to transmitter and receiver section. It is utilized for getting channel state information in the receiver side. After appending the pilots bit Serial to parahel converter is used for making 2 strands of data. The output of Serial to Parallel converter is fed into the IFFT block for OFDM modulation. Number of subcarrier equals the number of sub channels created by the $\mathrm{N}_{t} X \mathrm{~N}_{\mathrm{r}}$ MIMO system. The OFDM symbols generated are

$$
\mathbf{O}_{k}=\left\{\mathbf{o}_{k}{ }^{(0)}, \mathbf{o}_{\mathrm{k}}{ }^{(1)}, \mathbf{o}_{\mathrm{k}}{ }^{(2)}, \ldots \ldots \ldots \ldots \ldots . . . \mathbf{o}_{\mathrm{k}}{ }^{(\mathrm{Nt}-1)}\right\}
$$

where $\mathrm{O}_{\mathrm{k}}{ }^{(\mathrm{i})}$ denotes $\mathrm{OFDM}$ symbol; transmitted from $\mathrm{i}^{\text {th }}$ antenna through the subcarrier. In similar fashion the other users also send data through two transmitter antennas. The basic block diagram of transmitter section is shown in Figure 1. 


\section{Channel Model}
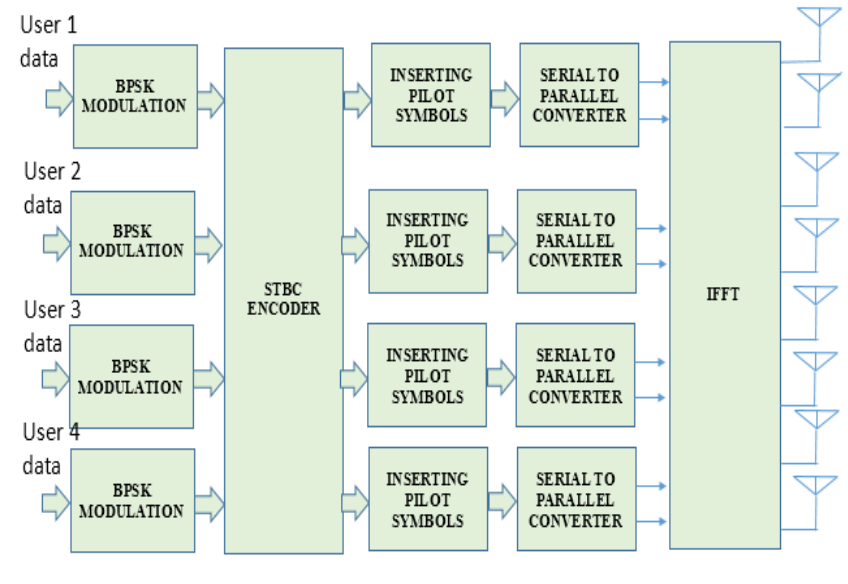

Figure 1. Transmiter Section

In this paper AWGN MIMO channel is Considered. Amplitude distortion, diffraction, shadowing and reflection from the surrounding objects, which arises due to multipath propagation, will affect the data transmitted through the channel [8]. Thus, the corrupted data arriving at the receiver antennas wilbe with different phase, amplitude and at different time interval.

Time domain response of MIMO channel from $\mathrm{i}^{\text {th }}$ transmitter antenna to $\mathrm{j}^{\text {th }}$ receiver antenna can be modeled by the equation as follows

$$
\mathbf{h}_{\mathrm{i}, \mathrm{j}}(\mathbf{t})=\sum_{l=0}^{L-1} \propto_{i, j}(\boldsymbol{l}) \boldsymbol{\delta}\left(\boldsymbol{t}-\tau_{l}\right)
$$

where $\propto_{i, j}$ is the multipath gain coffficient. Here L denotes the number of resolvable paths

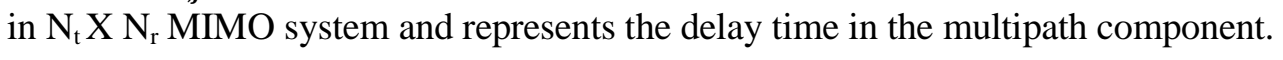

\section{Receiver Model}

The received OFDM symbol $\left\{\widehat{O_{K}}\right\}$ at the receiver antenna are passed through OFDM demodulator DFT will be performed since it mathematically similar OFDM demodulation operation. This will help to obtain encode symbols $\left\{\widehat{C_{K}}\right\}$ from the pilot symbols, known to both transmitter and receiver. The channel estimation is performed with the channel state information matrix. STBC decoding is performed to obtain the BPSK symbols

$$
\widehat{S}=\left\{\widehat{S_{0}}, \widehat{S_{1}}, \widehat{S_{2}}, \ldots \ldots, \widehat{S_{N-1}}\right\}
$$

Those symbols are passed through BPSK demodulator to obtain the bit stream

$$
\widehat{B}=\{\widehat{b 0}, \widehat{b 1}, \widehat{b 2}, \ldots \ldots b \widehat{b-1}\}
$$

The bit stream obtained is compared with input stream for varying SNR of channel 


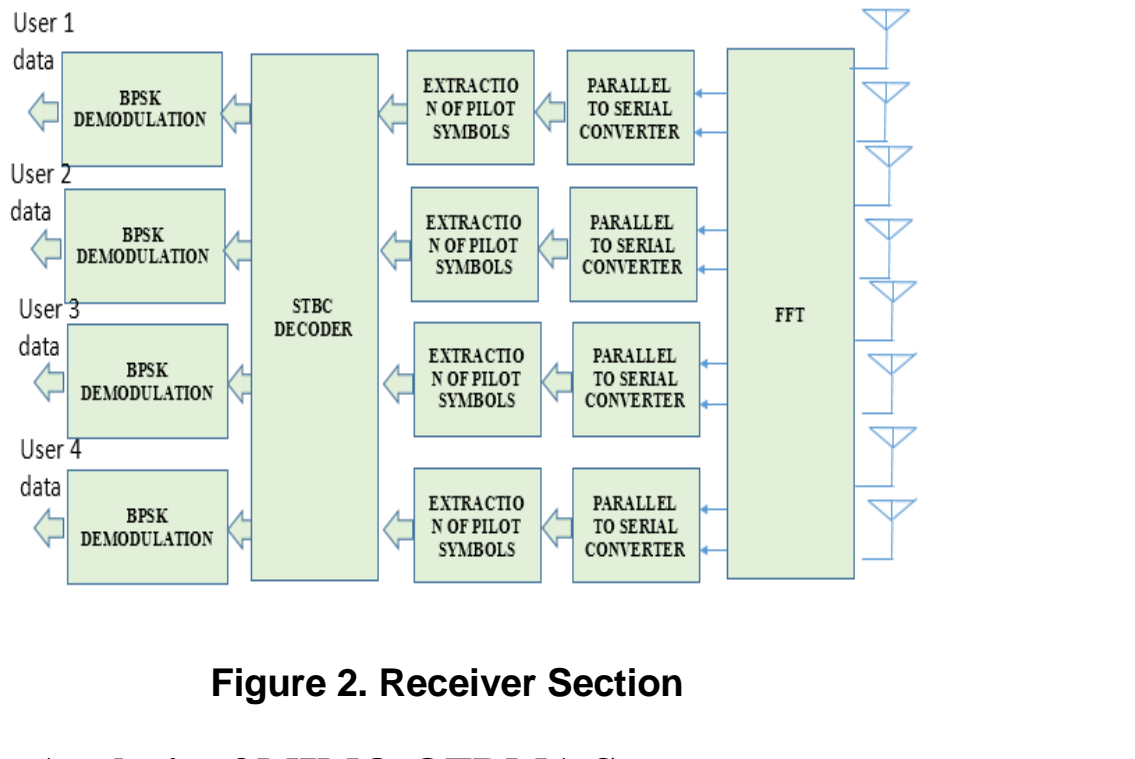

\section{Performance Analysis of MIMO-QfDMA System}

Performance analysis is investigated for MMMO OFDM system with both spatial diversity and multiplexing techniques and with spatial multiplexing alone. This is measured in the form of metric like BER and SER as a funetion of SNR of MIMO channel. The plots obtained from simulations are show in Figure 3 BER Plot Companison, which shows the values of BER as a function of SNR. Similarly, Figure 4 SER Rlot Comparison provides the plot for SER as a function of SNR. Both the flot provides better performance for both Spatial Diversity and Spatial Multiplexing implemented systemthan with spatial multiplexing alone.

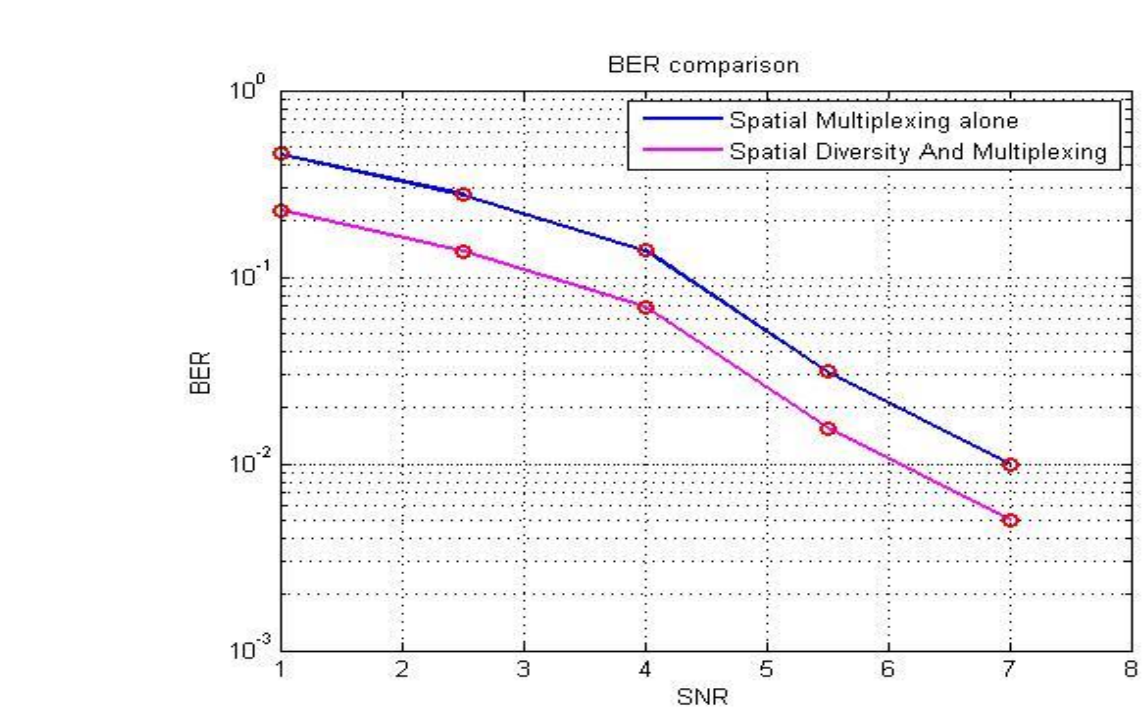

Figure 3. BER Plot Comparison 


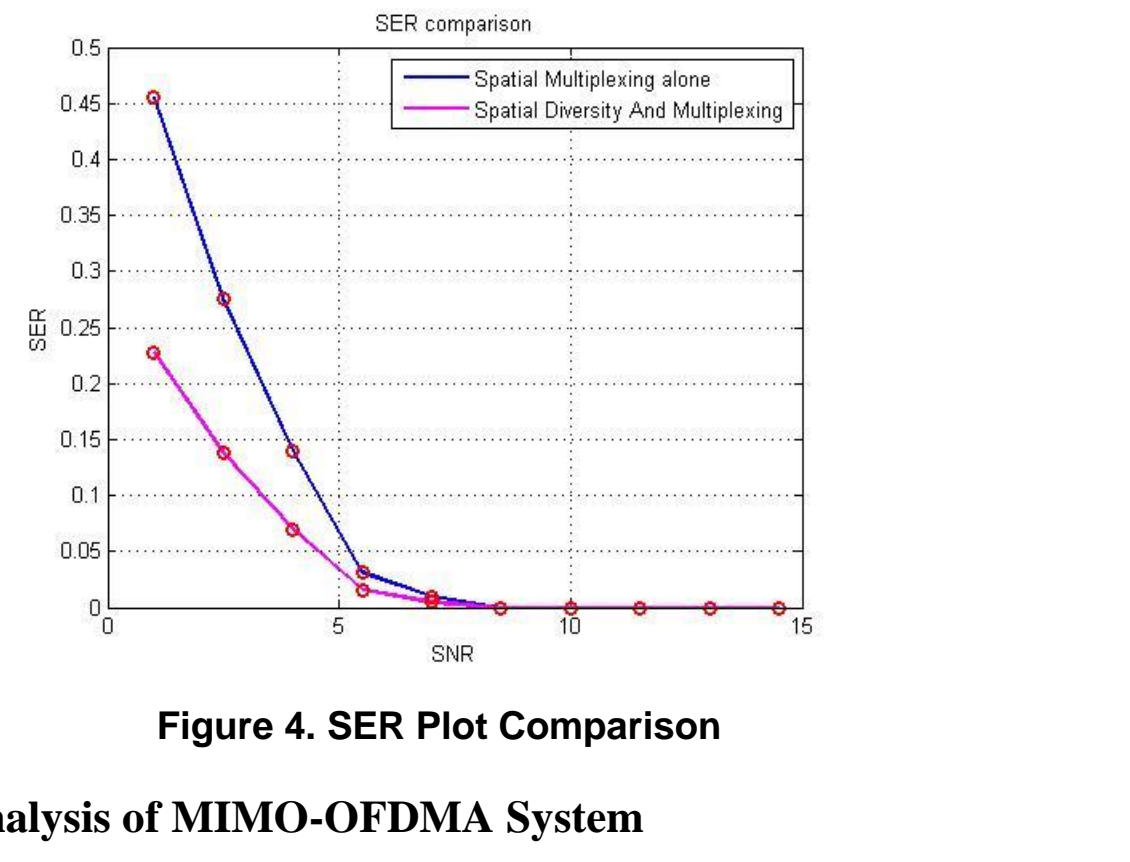

\section{Capacity Analysis of MIMO-OFDMA System}

Here MIMO channel capacity per unit bandwidth and throughput is investigated as a function of SNR. MIMO OFDM system with spatial diversity alone and with both spatial diversity and multiplexing techniques is compared for its spectral efficiency. The Figure 6 demonstrates the spectral effictency of MIMQ-OFDMA communication system with spatial diversity alone and with both spatial diversityand spatial multiplexing. The plots obtained from simulations are show in Elgure.5 Throughput Plot Comparison, which shows the values of Throughput as a function of SNR. Similarly, Figure.6 Spectral Efficiency Plot Comparison provides the plot for Spectral Efficiency as a function of SNR. Both the plot provides better performance for both Spatial Diversity and Spatial Multiplexing implemented system than with spatial multiplexing alone.

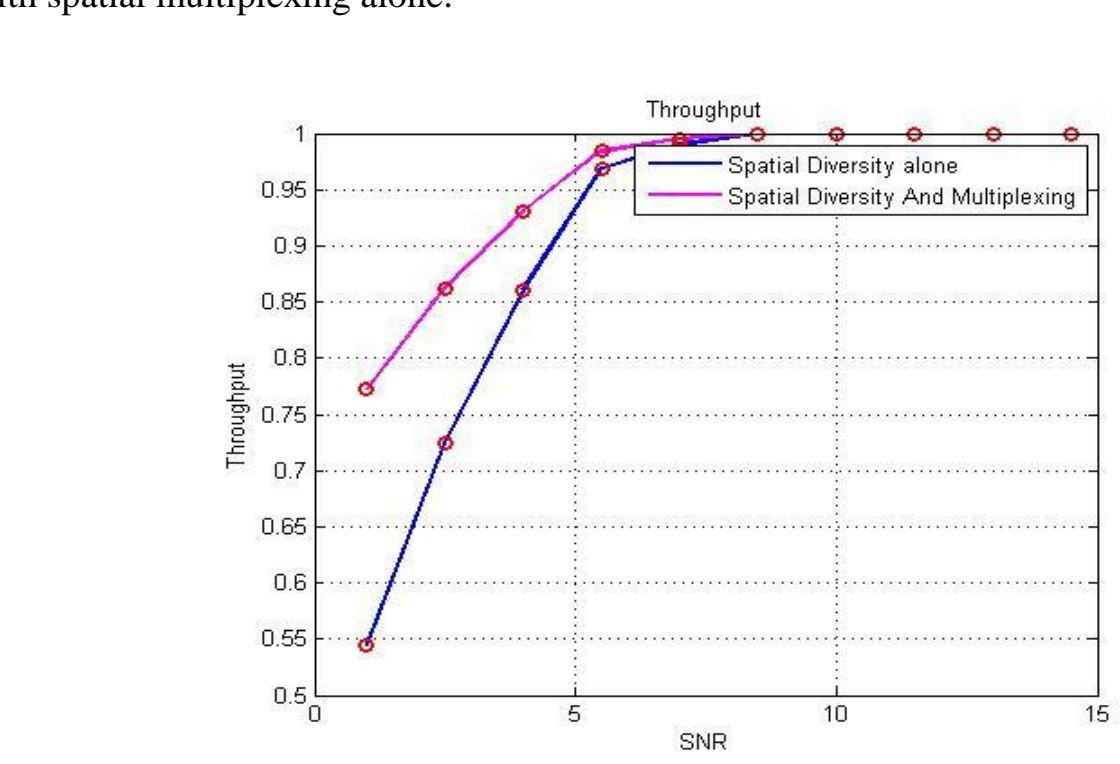

Figure 5. Throughput Plot Comparison 


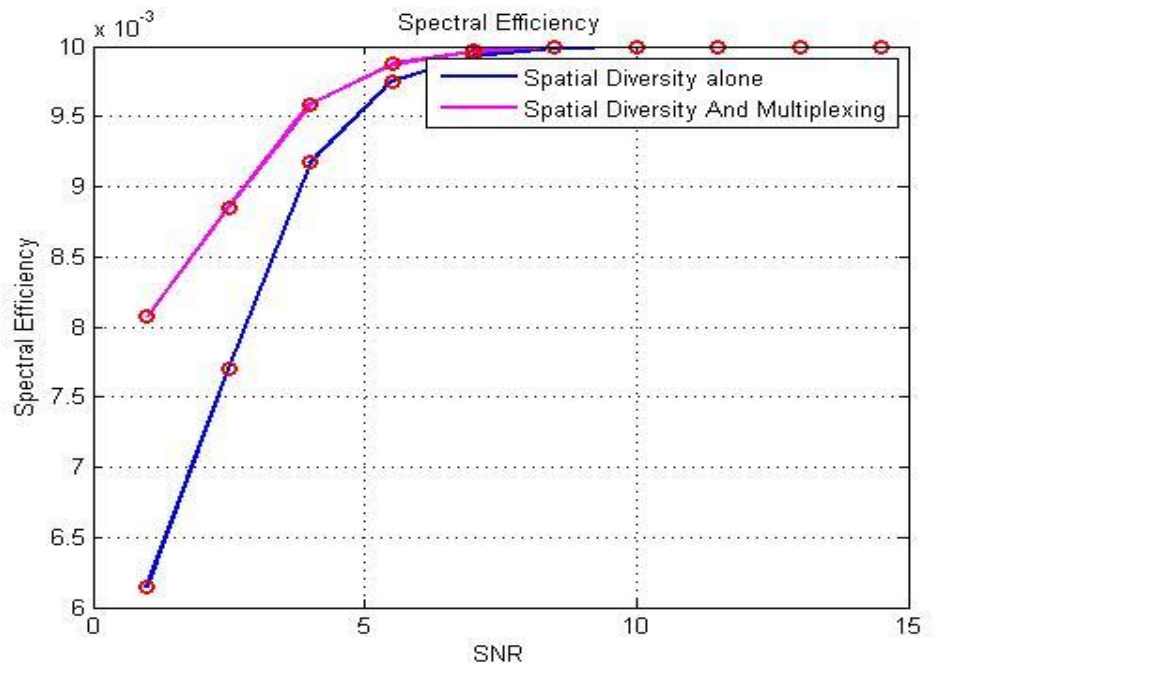

Figure 6. Spectral Efficiency Plot Comparison

\section{Conclusion}

In this paper the performance of the MMO-OFDMA system under AWGN channel is evaluated. Here the performance and capaeity of the system with spatial diversity alone and with both spatial diversity and spatial multwpexing are investigated for metrics like throughput and spectral efficiency as a function of \$NR. Similarly, performance and capacity of the system with spatial multiplexing alone and with both spatial diversity and spatial multiplexing are investigated for metrics iike BER and SER as a Function of SNR. From the analysis it is found that Spatial Multiplexing and Spatial Diversity implemented system is showing better performance metric dompared to with that of spatial multiplexing alone or spatial diversityalone

\section{References}

[1] J. Leinonen, J. Hamalainen and M. Juntti, "Senior Member, "Capacity Analysis of Downlink MIMOOFDMA Resource Allocation with Limited Feedback", IEEE IEEE transactions on communications, vol. 61, no. 1, (2013) Japuary.

[2] M. Morocco. "Performance and Capacity Analysis of Compact MIMO Aided OFDM-SDMA" Systems Progress In Electromagnetics Research Symposium Proceedings, (2011) March.

[3] I. Khan S.A. K. Tanoli and N. Rajatheva, "Capacity and performance analysis of space-time block coded MIMO-OFDM systems over Rician fading channel”, International Journal of Electrical and Computer Engineering (2009).

[4] R. H. Chen and W.-H. Chung, "Reduced Complexity MIMO Detection Scheme Using Statistical Search Space Reduction" IEEE COMMUNICATIONS LETTERS, vol. 16, no. 3, (2012) March.

[5] A. Burg, S. Haene, D. Perels, P. Luethi, N. Felber and W. Fichtner, "Integrated Systems Laboratory", ETH Zurich, Switzerland Texas A M University "Algorithm and VLSI Architecture for Linear MMSE Detection in MIMO- OFDM Systems. IEEE Xplore (2009) March.

[6] C.-Y. Hung and W.-H. Chung “An Improved MMSE-Based MIMO Detection using Low-Complexity Constellation Search" IEEE Globecom, Workshop on Broadband Wireless Access (2010).

[7] Md. M. Haque, M. S. Rahman and K.-D. Kim, "Performance Analysis of MIMO-OFDM for 4G Wireless Systems under Rayleigh Fading Channel", International Journal of Multimedia and Ubiquitous Engineering vol. 8, no. 1, (2013) January. 